\title{
Use of Functional Movement Screening to Determine Gender Differences in Flexibility and Injury Risk
}

ISSN: 2577-1914

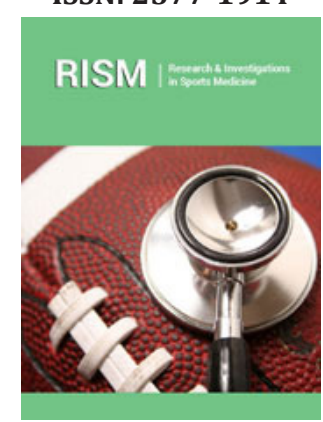

*Corresponding author: Taylor $\mathrm{M}$, Department of Kinesiology, University of the Sciences, Philadelphia, USA

Submission: 㘹 August 30, 2019

Published: 毕October 03, 2019

Volume 5 - Issue 3

How to cite this article: Taylor $M$, Moorman L, Marszalek A, Albur T, Dohm $\mathrm{H}$ and Thomas $\mathrm{E}$. Use of Functional Movement Screening to Determine Gender Differences in Flexibility and Injury Risk. Res Inves Sports Med. 5(3). RISM.000613.2019.

DOI: $10.31031 /$ RISM.2019.05.000613

Copyright@ Taylor M, This article is distributed under the terms of the Creative Commons Attribution 4.0 International License, which permits unrestricted use and redistribution provided that the original author and source are credited.

\author{
Taylor M*, Moorman L, Marszalek A, Albur T, Dohm H and Thomas E \\ Department of Kinesiology, Philadelphia, USA
}

\begin{abstract}
Each year in the United States an estimated 4.5 million sports-and recreation-related injuries occur in children and young adults [1]. With more young adults participating in sport and more injuries, there has been some research on prevention and treatment of injuries. However, research has not been done on a comprehensive screening to determine which athletes are more susceptible. The Functional Movement Screen (FMS) was developed as a pre-participation screen to reflect the combination of strength, flexibility, range of motion, coordination, and balance. Few studies have formally investigated the use of the FMS in male and female athletes to predict injury risk and compare differences between genders $[2,3]$.
\end{abstract}

Purpose: To test and compare college male and female basketball player FMS scores and track injuries during the competitive season in order to determine if low FMS scores correlate with higher injury rates.

Methods: 19 female (age $19.3 \pm 0.23$ ) and 17 male (age 20.6 \pm 0.31 ) collegiate basketball players participated in this investigation. Participants engaged in one session of FMS testing during pre-season training. All seven movement patterns were tested and scored. Injuries were tracked and scored based on severity T-test were used to examine the relationship between FMS score, gender, and injury rates.

Student's t-tests were used to determine significant differences between male and female athletes.

Results: For several movements, females scored significantly higher on the FMS screening than the males $(\mathrm{p}<.05)$. Analysis showed no significant correlation found in either group (R2=.36 for males and .01 for females)

Conclusion: Results indicate that female athletes scored significantly higher on the FMS. The relationship between FMS score and injury rate remains unclear. The male players with low FMS scores trended towards having a higher injury score. This may suggest that males with a lower FMS score are at a higher risk for injury. For females, the same trend was not observed. Further studies are required to determine the role of this test in determining injury susceptibility and the utility of the FMS for males versus females.

\section{Introduction}

Each year in the United States an estimated 4.5 million sports- and recreation-related injuries occur in children and young adults [1]. Sports are intended to provide individuals with many positive physical health benefits, as well as emotional and social benefits, but the risk of injury produces unwanted negative effects. Injury is a serious concern for all agesfrom children just starting to professionals, and all ages in between. The number of female athletes involved in sports has increased following Title IX, but the injury rates in females have also increased dramatically. According to the National Federation of State High School Associations, participation in high school girls' sports has increased from 294,000 in 1971 to nearly 3.2 million in 2011 [4]. Female athletes are injured more than their male counterparts - as much as two to six times more depending on the sport [5]. While it is impossible to completely eliminate athletic injuries, one can look at measures to predict future injury occurrences. Being able to predict injuries would allow for better prevention strategies, reducing the negative effects athletes experience as a result of sports.

With more children and teenagers participating in sport and more injuries, there has been some research on prevention and treatment of injuries. However, research has not been done on a comprehensive screening to determine which athletes are more susceptible. Many studies focus on a specific joint, such as Devan, Pescatello, Faghri, \& Anderson's 2004 study 
based only on injuries to the knee, and don't look at the whole body or other factors that could play a role in injury [6]. Many factors play a role in musculoskeletal injuries. Previous epidemiologic studies have noted sedentary lifestyles, smoking, gender and prior injuries as major risk factors [1]. A variety of other intrinsic factors predisposing athletes to injury have also been documented in the literature, including body composition, fitness level, bone structure, muscle strength, age, and gender [7].

In athletic populations, muscle imbalances and decreased range of motion can lead to more injuries. Previous studies have looked at the relationship between strength and flexibility imbalances and structural abnormalities in the preseason on athletic injuries in collegiate athletes. However, much of the published literature on risk factors for injuries in sports have been retrospective. In an effort to bridge the gap between required pre-participation medical screening and optional performance testing, Cook developed the Functional Movement Screen (FMS) [8,9]. The FMS consists of seven movement tests intended to quickly and easily identify restrictions or alterations in normal movement. The FMS is able to target and to identify inefficient movement strategies by noting compensations or imperfections in the movement tests [8]. This screening method provides a comprehensive evaluation rather than having to focus on specific factors such as quadriceps to hamstring ratios or other structural imbalances.

Compensations in fundamental movements lead to inefficient movements that could put the individual at a greater risk of injury. This is especially prevalent in today's athletics because athletes are starting at such a young age and are oftentimes specializing early on in their careers. If the athlete's movement pattern is not corrected early on, years of repetitive movements in his or her sport can lead to the continuation of functional movements that are not biomechanically sound and may place added stress on the body [10]. By adopting these inefficient movement strategies, individuals may reinforce poor movement patterns that, despite achieving high performance, may eventually result in injury [2]. The goal of FMS is to be able to discover these inefficient movement patterns in order to predict and prevent future injury. A review of the literature highlighted the potential of FMS as a new screening tool, including the ability of FMS to measure fundamental movements, prediction leading to prevention, and the practicality of FMS.

Injuries in this population are a major concern that has gained a lot of attention over the years since Title IX resulted in an increased number of female athletes. If low scores on the FMS can predict these injuries, it can lead to better prevention strategies. This knowledge would be helpful for strength and conditioning coaches, athletic trainers, and coaches to provide awareness and education. Finding low scores or weaknesses in specific areas would enable those in the sports medicine field to add "pre-hab" into the program design by strengthening weak muscles and stretching those areas that lack flexibility. Coaches and trainers have a limited time with the athletes, so it is important to have a practical and quick way to predict injury rates and prevent injuries.
FMS testing can quickly determine how an individual performs everyday functional movements, and it could easily be implemented into a pre-season evaluation in many sports [9]. Few studies have formally investigated the use of the FMS and its ability to predict injury in the athletic population in general, and it is especially limited in the female athletic population. The purpose of this study is to determine if compensatory movement patterns, as measured by FMS, predispose collegiate basketball players to injury, and if Gray Cook's Functional Movement Screen could be used to measure those inefficient movements in order to predict injury in this population over the course of one competitive season. It is hypothesized that FMS scores will be inversely correlated with the number of injuries as well as severity of injuries. As well as analyzing gender differences in FMS score and injury rates related to those scores.

\section{Methods}

In this study, FMS testing was done during a pre-season basketball practice. The collegiate athletes were players on the men's and women's basketball team at a small private university in the Midwest. Both head coaches were informed of the study. They were provided with a brief overview of the purpose, clinical significance, anticipated outcomes, and practical application of this investigation. The primary investigator announced the study to team members at St. Ambrose explaining the testing procedure and necessary disclosure of athletic injuries. All participation was strictly voluntary. Decision to participate in the study had no bearing on eligibility status or interfered with practice time. The potential risks of the Functional Movement Screen for participants were minimal to no due to the screening consisting of normal everyday functional movements. The Institutional Review Board at St. Ambrose University approved the study.

Participants were asked to perform a series of movements using directions for testing as described by the authors of the FMS [9]. The FMS consists of seven movement tests, described by Cook et al. [8] that include: Deep Squat, Hurdle Step, In-Line Lunge, Shoulder Mobility, Active Straight Leg Raise, Trunk Stability Push-Up, and Rotary Stability. The athletes were instructed on how to perform each movement, and the investigator demonstrated each test prior to the athlete completing the test. Each of the seven fundamental movement tests are scored from 0 to 3 -a score of 3 indicates the individual was able to perform the movement correctly, 2 indicates compensation or a modification required to complete the movement, 1 shows the individual was not able to perform the movement, and 0 is representative of either being unable to assume the starting position or the individual experienced pain during the movement [8].

Injuries sustained by each participant during in-season practices and competitions were then reported in weekly followups with the certified athletic trainers overseeing the collegiate basketball team. The definition of injury utilized for the purpose of this study was a musculoskeletal injury that meets the following criteria: 
1. The injury occurred as a result of participation in an organized intercollegiate practice or competition setting and

2. The injury required medical attention, or the athlete sought advice from a certified athletic trainer. Injuries were then scored based on the length of time the athlete was out of practice/ games. Minor injuries that kept players out of practice/games for no more than one day were scored as a one, injuries that caused players to miss 2-6 days were given a two, and those that kept players out for a week or more were scored as a three. The primary researcher, utilizing information from the injury reports received, determined these scores. After injuries were scored and recorded, t-tests were used to examine any differences between the men's and women's team. FMS results were analyzed using a t-test to examine patterns in FMS scores among the male and female athletes. T-tests were also performed to compare FMS scores for individual components of the between the males and females.

\section{Result}

This study also examined the relationship between FMS score and patterns of injury in male and female athletes. Table 1 presents the participant demographics, average FMS scores, and average injury scores. When examining the injuries occurring among male and female basketball players, no significant differences were found, indicating that neither sex is more likely to suffer an injury during the course of the season. In general, there were few injuries among both groups.

FMS scores were collected prior to the start of the athletic season. This test was performed on both the male and female athletes. The average scores for each movement are shown in Figure 1. The difference in total FMS score between male and female athletes was found to be statistically significant $(\mathrm{p}<.05)$ with females averaging $16.4 \pm 0.41$ out of a possible high score of 21 and the males averaging $15.2 \pm 0.37$ (Table 1 ).

In an examination of differences between various movements, female athletes scored significantly $(\mathrm{p}<.05)$ higher on both shoulder mobility tests ( 2.47 for the females compared to 1.76 for the males for the right and 2.84 for females versus 2.11 for males for the left) and the deep squat (2.26 for females and 2 for males (Figure 1 ). It should also be noted that females had significantly higher overall FMS scores despite males having scored significantly higher on the Trunk Stability test when compared to the females.

For the female athletes, seven injuries were recorded during the competitive basketball season. There were six recorded injuries for the male athletes. Injuries were divided into categories based on the location of the injury and scored based on severity. Figures $2 \& 3$ examined the results of individual injury type and total injury score. There were no statistically significant differences in injury scores between the female and male athletes.

\section{Discussion}

Results indicate that female athletes scored significantly higher on the FMS. The relationship between FMS score and injury rate remains unclear. There was no significant relationship between low FMS scores and higher injury rates or between low FMS scores and injury severity. This is not consistent with results discussed by Chorba et al. [2] and O'Connor et al. [7] which showed FMS quantifies inefficient movement patterns and low scores result in a higher rate of injuries [2,7]. The study also showed that there were no differences in injury rates between men and women, and there were significant differences in FMS scores. There were significant differences shown in FMS scores for deep squats, shoulder mobility, and push-ups. There were significant differences with men scoring higher on push-ups versus women scoring higher on deep squat and shoulder mobility. The significance in these exercises could be less related to injury than other exercises and mobility test.

A possible limitation of this study is the small sample size. The majority of athletes scored at least a 15 on the FMS, so there was a restricted range of scores. Previous research suggests 14 is a critical score at which injury rates increase significantly [2]. In this study, only three athletes scored at or below 14; two of which became injured. Repeating this research with a larger sample would be beneficial, as only a few injuries were recorded and studied. The injury reports received by the primary investigator contained minimal information regarding how the injury occurred. In the future, each individual injury could be looked into further in order to understand the mechanism of injury better [11-16].

Future research could look further into specific tests within the Functional Movement Screen to determine if one of the seven individual tests is able to predict specific injuries. Also, future research could be done on athletes who have had previous injuries and/or surgeries to determine how procedures, such as ACL reconstruction, affect scores on the FMS. More research will need to be done in order to evaluate how FMS testing predicts injuries in any other sports. Studies could be done on athletes, male and female, in other sports that may be more upper body dominant in order to determine how useful FMS is at predicting injuries. In addition, one could research how different positions within a sport compare regarding FMS score and injury. Additional research on the use of FMS as an injury predictor could aide athletic trainers and coaches.

\section{References}

1. Fernandez WG, Yard EE, Comstock RD (2007) Epidemiology of lower extremity injuries among US high school athletes. Acad Emerg Med 14(7): 641-645.

2. Chorba RS, Chorba DJ, Bouillon LE, Overmyer CA, Landis JA (2010) Use of functional movement screening tool to determine injury risk in female collegiate athletes. North American Journal of Sports Physical Therapy 5(2): 47-54.

3. Warren M, Lininger MR, Chimera NJ, Smith CA (2018) Utility of FMS to understand injury incidence in sports: current perspectives. Open Access Journal Of Sports Medicine 9: 171-182.

4. Flanagan L, Greenberg S (2012) How Title IX Hurts Female Athletes.

5. Harber V (2010) Female athletes injured more than male athletes.

6. Devan M, Pescatello L, Faghri P, Anderson J (2004) A prospective study of overuse knee injuries among female athletes with muscle imbalances and structural abnormalities. J Athl Train 39(3): 263-267. 
7. O'Connor FG, Deuster PA, Davis J, Pappas CG, Knapik JJ (2011) Functional movement screening: Predicting injuries in officer candidates. Med Sci Sports Exerc 43(12): 224-2230.

8. Cook G (2010) Movement: Functional movement systems: Screening, assessment, and corrective strategies. On Target Publications, Aptos, California, USA

9. Cook G, Burton L, Hoogenboom B (2006) Pre-participation Screening: The use of fundamental movements as an assessment of function - Part 1. North American Journal of Sports Physical Therapy 1(2): 62-72.

10. Brenner J (2007) Overuse injuries, overtraining, and burnout in child and adolescent athletes. Pediatrics 119(6): 1242-1245.

11. Duncan MJ, Stanely M (2012) Functional movement is negatively associated with weight status and positively associated with physical activity in British primary school children. Journal of Obesity.
12. Hasan FF, Ibrahim EK (2012) The effect of gender on Range of Motion (ROM) for knee joint. Tikrit Medical Journal 18(1): 112-120.

13. Kiesel K, Plisky PJ, Voight ML (2007) Can serious injury in professional football be predicted by a preseason functional movement screen? North American Journal of Sports Physical Therapy 2(3): 147-158.

14. Okada T, Huxel KC, Nesser TW (2011) Relationship between core stability, functional movement, and performance. Journal of Strength and Conditioning Research 25(1): 252-261.

15. Parchman CJ, McBride JM (2011) Relationship between functional movement screen and athletic performance. Journal of Strength \& Conditioning 25(12): 3378-3384.

16. Schneiders AG, Davidson A, Horman E, Sullivan J (2011) Functional movement screen normative values in a young, active population. International Journal of Sports Physical Therapy 6(2): 75-82. 